\author{
JORGE J. GUMUCIO, EDITOR \\ Department of Internal Medicine \\ Division of Gastroenterology (111D) \\ VA Medical Center/University of Michigan \\ Ann Arbor, Michigan 48105
}

\section{CHOLANGITIS AND ENDOSCOPIC DRAINAGE}

Lai ECS, Mok FPT, Tan ESY, Lo C-M, Fan S-T, You K-T, Wong J. Endoscopic biliary drainage for severe acute cholangitis. N Engl J Med 1992;326:1582-1586.

\section{ABSTRACT}

Background. Emergency surgery for patients with severe acute cholangitis due to choledocholithiasis is associated with substantial morbidity and mortality. Because recent results suggested that emergency endoscopic drainage could improve the outcome of such patients, we undertook a prospective study to determine the role of this procedure as initial treatment.

Methods. During a 43-month period, 82 patients with severe acute cholangitis due to choledocholithiasis were randomly assigned to undergo surgical decompression of the biliary tract (41 patients) or endoscopic biliary drainage (41 patients), followed by definitive treatment. Hospital mortality was analyzed with respect to the use of endoscopic biliary drainage and other clinical and laboratory findings. Prognostic determinants were studied by linear discriminant analysis.

Results. Complications related to biliary tract decompression and subsequent definitive treatment developed in 14 patients treated with endoscopic biliary drainage and 27 treated with surgery ( 34 vs. 66 percent, $P>0.05$ ). The time required for normalization of temperature and stabilization of blood pressure was similar in the two groups, but more patients in the surgery group required ventilatory support. The hospital mortality rate was significantly lower for the patients who underwent endoscopy (4 deaths) than for those treated surgically (13 deaths) (10 vs. 32 percent, $P<0.03)$. The presence of concomitant medical problems, a low platelet count, a high serum urea nitrogen concentration, and a low serum albumin concentration before biliary decompression were the other independent determinants of mortality in both groups.

Conclusions. Endoscopic biliary drainage is a safe and effective measure for the initial control of severe acute cholangitis due to choledocholithiasis and to reduce the mortality associated with the condition.

\section{COMMENTS}

Acute cholangitis (classic diagnosis triad of jaundice, fever/chills and right upper quadrant pain) may respond to antibiotics, allowing later elective stone removal. However, severe "suppurative" cholangitis (classic diagnostic pentad including hypotension and mental status changes) historically carries a $100 \%$ mortality

31/8/41747 rate without urgent surgical decompression $(1,2)$. Even with modern surgical techniques and antibiotics, surgical mortality rates remained greater than $20 \%$ in the 1980 s $(3,4)$. Recently, endoscopic techniques have been successfully applied to the therapy of severe acute cholangitis as they have been to a variety of other "surgical" diseases during the last several years (4-8). This study by Lai et al. from Hong Kong is the first to compare emergent surgical decompression of the biliary tract to endoscopic biliary drainage in a controlled prospective manner.

The diagnosis of severe acute cholangitis was based on symptoms of cholangitis plus either septicemic shock or progressive biliary infection including mental confusion and persistent fevers despite antibiotics. Randomization occurred just after diagnostic endoscopic cholangiography (ERC) was performed. The endoscopic drainage patients received was a small papillotomy and placement of a 7F nasobiliary catheter, with no attempt to remove the stones. Later, after symptoms subsided, definitive surgical or endoscopic therapy was completed. Patients randomized to surgery underwent emergent common bile duct exploration under general anesthesia a mean of $2 \mathrm{hr}$ after the diagnostic ERC.

Might injection of contrast into an infected, pressurized biliary system combined with the delay to surgery (albeit short) have contributed to the increased septic complication rate in the surgical group? Probably not. Although two patients had shock develop after ERC while awaiting surgery, the duration of hypotension before surgery or endoscopic drainage was the same in both groups. The mean systolic blood pressure before drainage was actually higher in the surgical group. Not surprisingly, the surgical group had a higher incidence of wound infections/dehiscence ( 8 vs. 1 ) and bronchopneumonia (15 vs. 7) than the endoscopic group. These accounted for most of the postprocedure complications. Thus the surgical procedure itself and not the time delay from ERC to surgery appeared to account for the doubled morbidity and tripled mortality rates seen in these patients compared with those treated with endoscopic drainage.

Are the results of this study applicable to the Western population? Probably yes. Lai's group identified 96 patients with severe acute cholangitis ( 14 were excluded) out of nearly 500 patients with acute cholangitis in just over $3.5 \mathrm{yr}$. These large numbers are undoubtedly due in part to the high incidence of Oriental cholangiohepatitis (recurrent pyogenic cholangitis) in Hong Kong. This disease represents the third most common abdominal cause of emergency admissions to Hong Kong hospitals 
(9). Unlike most ductal stones in the West, which are secondary cholesterol stones and are seen in a predominantly elderly, female population, the stones of Oriental cholangiohepatitis are soft calcium bilirubinate stones, are found in a younger population and occur with equal incidence in both men and women. The etiological role of chronic parasitic (Clonorchis sinensis, Ascaris lumbricoides) and bacterial infections in intrahepatic stone formation in this disease remains arguable. Recurrent attacks of cholangitis are the rule; most have intrahepatic ductal stones, and biliary strictures are found in one third $(10,11)$. Although Lai's population shows an equal sex distribution and the frequency of recurrent cholangitis is not detailed, some features suggest a similarity to the Western population. These include an older age group (mean age $=69 \mathrm{yr}$ ) and a relatively low incidence of intrahepatic ductal stones $(10 \%)$ and biliary strictures $(10 \%)$. The conclusion drawn by this study would likely apply to all populations. Less complicated ductal stone disease characteristic of the Western population, if anything, would further favor the endoscopic group because rapid stone extraction may be possible at the time of initial ERC, thereby avoiding follow-up procedures.

Endoscopic drainage procedures that may be considered instead of nasobiliary drainage include stent placement and simple sphincterotomy alone. Sphincterotomy alone may provide adequate drainage and even ductal clearance when stones are small and few in number, but large stones and chunks of debris will cause persistent obstruction. Stent placement is comparable to nasobiliary insertion. Stenting offers the advantage of a prosthesis that is completely internal and therefore is not accidentally dislodged by the patient. It is easily maintained for weeks or months if necessary. Alternatively, a nasobiliary tube is easily flushed to prevent clogging and may be injected for repeat cholangiography. The choice of endoscopic drainage procedure will be determined by the extent of choledocholithiasis, the anticipated time and need for follow-up procedures, anatomic technical considerations and physician preference.

Is percutaneous drainage a viable option in this patient population? Of course it is. Local availability and expertise should be the major determinant of the type of drainage technique used. Percutaneous biliary drainage was not examined in this study, but others have found variable outcomes after this procedure in uncontrolled reports $(12,13)$.

Severe acute cholangitis with sepsis develops when bacteria from infected bile under pressure enter the bloodstream. Biliary drainage and antibiotic administration must be performed. Now it is clear that this is better accomplished endoscopically rather than surgically in the emergent situation.

JEFFREY L. BARNETT, M.D. Gastroenterology Division University of Michigan Medical School Ann Arbor, Michigan 48109-0362

\section{REFERENCES}

1. Leung JWC, Chung SCS, Sung JJY, Banez VP, Li AKC. Urgent endoscopic drainage for acute suppurative cholangitis. Lancet 1989;1:1307-1309.

2. Andrew DJ, Johnson SE. Acute suppurative cholangitis: a medical and surgical emergency. Am J Gastroenterol 1970;54:141-154.

3. Lai ECS, Tam PC, Paterson IA, Ng MMT, Fan ST, Choi TK, Wong J. Emergency surgery for severe acute cholangitis; the high-risk patients. Ann Surg 1990;211:55-59.

4. Leese T, Neoptolemos JP, Baker AR, Carr-Locke DL. Management of acute cholangitis and the impact of endoscopic sphincterotomy. Br J Surg 1986;73:988-992.

5. Delmotte JS, Pommelet $P$, Houcke $P$, et al. Initial duodenoscopic sphincterotomy in patients with acute cholangitis or pancreatitis complicating biliary stones [Abstract]. Gastroenterology 1982;82: 1042.

6. Gogel KH, Runyon BA, Volpicelli NA, Palmer RC. Acute suppurative obstructive cholangitis due to stones: treatment by urgent endoscopic sphincterotomy. Gastrointest Endose 1987;33: 210-213.

7. Ikeda S, Tanaka M, Itoh H, Kishikawa H, Nakayama F. Emergency decompression of bile duct in acute obstructive suppurative cholangitis by duodenoscopic cannulation: a lifesaving procedure. World J Surg 1981;5:587-593.

8. Lai ECS, Paterson IA, Tam PC, Choi TK, Fan ST, Wong J. Severe acute cholangitis: the role of emergency nasobiliary drainage. Surgery 1990;107:268-272.

9. Li AKC, Chung SCS, Leung JWC, Mok SD. Recurrent pyogenic cholangitis: an update. Trop Gastroenterol 1985;6:119-131.

10. van Sonnenberg E, Casola G, Cubberley DA, et al. Oriental cholangiohepatitis: diagnostic imaging and interventional management. AJR 1986;146:327-331.

11. Chen HH, Zhang WH, Wang SS. Twenty-two year experience with the diagnosis and treatment of intrahepatic calculi. Surg Gynecol Obstet 1984;159:519-524.

12. Pessa ME, Hawkins IF, Vogel SB. The treatment of acute cholangitis: percutaneous transhepatic biliary drainage before definitive therapy. Ann Surg 1987;205:389-392.

13. Kinoshita H, Kirohaski K, Igawa S, Nagata E, Sakai K. Cholangitis. World J Surg 1984;8:963-969.

\section{SURGICAL MANAGEMENT OF THE BUDD-CHIARI SYNDROME: NO PLACE FOR A PROCRUSTEAN BED}

Bismuth $H$, Sherlock DJ. Portasystemic shunting versus liver transplantation for the Budd-Chiari syndrome. Ann Surg 1991;214:581-589.

\section{ABSTRACT}

Over 12 years, 22 patients with the Budd-Chiari syndrome were treated surgically. Eishteen underwent a mesenterico-caval shunt (MCS); two, a side-to-side portacaval shunt; one, a mesentericoatrial shunt (MAS); and one, a liver transplantation (OLT). One patient died after operation from the precipitating condition, and two MCS grafts that thrombosed were restored. All 21 surviving patients remain well, free from ascites, and all shunts are patent after a mean follow-up of $5.6 \pm 1$ years, five patients with more than 10 years' follow-up. This long-term survival achieved by portasystemic shunts suggests that they have a major role in the treatment of the Budd-Chiari syndrome. The authors prefer the mesenterico-caval shunt using a jugular graft. This ensures a total portasystemic shunt, avoids subhepatic surgery, and reduces the long-term risk of prosthetic graft throm- 